\title{
Innate Bone Remodeling
}

National Cancer Institute

\section{Source}

National Cancer Institute. Innate Bone Remodeling. NCI Thesaurus. Code C28754.

Innate Bone Remodeling takes place in the adult skeleton at discrete foci. The continuous turnover of bone matrix and mineral that involves first, an increase in bone resorption (osteoclastic activity) and later, reactive bone formation (osteoblastic activity). This process ensures the mechanical integ rity of the skeleton throughout life and plays an important role in calcium homeostasis. An imbalance in the regulation of two contrasting events, bone resorption and bone formation, results in many of the metabolic bone diseases, such as OSTEOPOROSIS. 